\title{
Simulated weightlessness procedure, head- down bed rest impairs adult neurogenesis in the hippocampus of rhesus macaque
}

\author{
Xu Zhang ${ }^{1 \dagger}$, Xixia Chu ${ }^{1 \dagger}$, Lei Chen ${ }^{1 \dagger}$, Juan Fu ${ }^{2 \dagger}$, Shuai Wang ${ }^{1}$, Jinjing Song ${ }^{1}$, Guanghan Kan ${ }^{3}$, Weizhong Jiang ${ }^{4}$, \\ Guang $\mathrm{He}^{1}$, Xiaoping Chen ${ }^{3^{*}}$ and Weidong $\mathrm{Li}^{\mathrm{i}^{*}}$
}

\begin{abstract}
The microgravity environment in space can impact astronauts' cognitive and behavioral activities. However, due to the limitations of research conditions, studies of biological changes in the primate brain, such as neurogenesis, have been comparatively few. We take advantage of $-6^{\circ}$ head-down bed rest (HDBR), one of the most implemented space analogue on the ground, to investigate the effects of weightlessness on neurogenesis of non-human primate brain. Rhesus Macaque monkeys were subjected to HDBR for 42 days to simulate weightlessness. BrdU (5-bromodeoxyuridin) and IdU (iododeoxyuridine) were intraperitoneally injected separately before or after HDBR to label the survival and proliferation of newborn neurons. Immunohistochemistry was performed to study the effect of simulated weightlessness on neurogenesis. BrdU staining showed that survival of newborn neurons was reduced, while there were fewer BrdUpositive neurons in the HDBR group compared with the control. Furthermore, IdU-positive neurons also decreased in the HDBR group suggesting a reduced proliferation capacity for these newborn neurons. Our results demonstrate the definite neurogenesis in the adult rhesus macaque hippocampus, and simulated weightlessness HDBR procedure impairs the adult neurogenesis.
\end{abstract}

Keywords: Adult neurogenesis, Simulated weightlessness, Rhesus monkey

There have been many long-duration spaceflights over the past decades, and more spaceflights with even longer durations will be required in the future. Humans, instead of robots, have an essential role in long-duration spaceflight missions due to superior perception, intelligent decisionmaking and capacity for independent action. It is clear that the microgravity environment in space can impact astronauts' cognitive and behavioral activities $[1,2]$, which further affects the astronauts' decision-making [3]. This could be noteworthy risk for long-duration spaceflight missions. Therefore, it is of great importance to reveal the underlying

\footnotetext{
* Correspondence: xpchen2009@163.com; liwd@sjtu.edu.cn

${ }^{\dagger}$ Xu Zhang, Xixia Chu, Lei Chen and Juan Fu contributed equally to this work.

${ }^{3}$ National Key Laboratory of Human Factors Engineering, China Astronaut Research and Training Center, Beijing 100094, China

${ }^{1}$ Bio-X Institutes, Key Laboratory for the Genetics of Development and Neuropsychiatric Disorders (Ministry of Education), Shanghai Key Laboratory of Psychotic Disorders, and Brain Science and Technology Research Center, Institute of Psychology and Behavioral Sciences, Shanghai Jiao Tong University, 800 Dongchuan Road, Shanghai 200240, China Full list of author information is available at the end of the article
}

mechanism of how microgravity leads to abnormal cognitive and behavioral activities. Physiology studies reveal that changes in volumes of cerebrospinal fluid, cerebral blood flow and intracranial pressure are caused by the redistribution of an astronaut's body fluid toward the head in a weightless environment $[4,5]$, and this may lead to structure remodeling. Neuroimaging studies have demonstrated alterations in the volumes of gray matter and white matter in specific brain regions including the frontal lobes and the hippocampus [6]. Early studies have also shown microgravity affects neurotransmitter concentrations [7] and the number of synapses [8].

Most studies on brain tissues under microgravity have been based on rat and mouse animal models [9], which might not simulate human activities well. Previous studies in human have reported the effect of spaceflight on psychological problems, cephalic fluid shifts, and cognitive alterations, however biological changes in the brain are not as well investigated [10]. There are few reports on whether brain abnormalities caused by space flight are related to

(c) The Author(s). 2019 Open Access This article is distributed under the terms of the Creative Commons Attribution 4.0 International License (http://creativecommons.org/licenses/by/4.0/), which permits unrestricted use, distribution, and 
neurogenesis. In the last decade, there is increasing evidence demonstrating the important role of adult hippocampal neurogenesis in the pathogenesis and therapeutics of mental diseases. Head-down bed rest (HDBR) is the widely used procedure to study the effects of simulated weightlessness on primates on the ground. HDBR eliminates gravitational input form the head to the leg and inducing cephalic fluid shift from the lower parts of the body toward the head by applying bed rest with $6^{\circ}$ head down position ([11], Fig. 1a and Additional file 1: Figure S1). However, the subjects are still under normal gravity during the HDBR procedure. With the advantage of HDBR animal model, we

\section{a}

\section{$-6^{\circ}$ head-down bed rest}

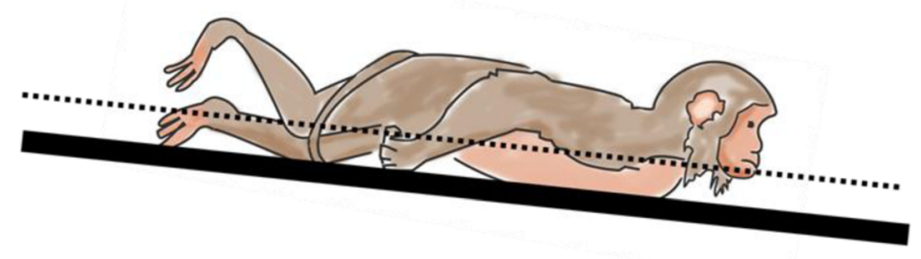

b

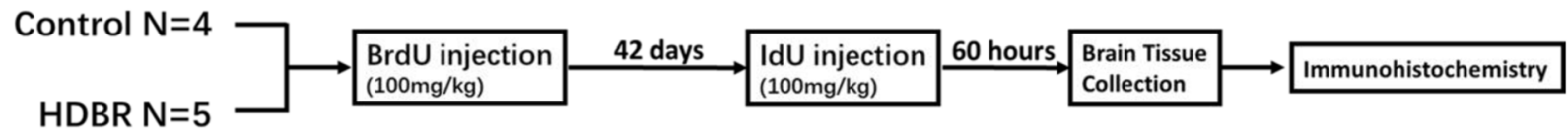

C
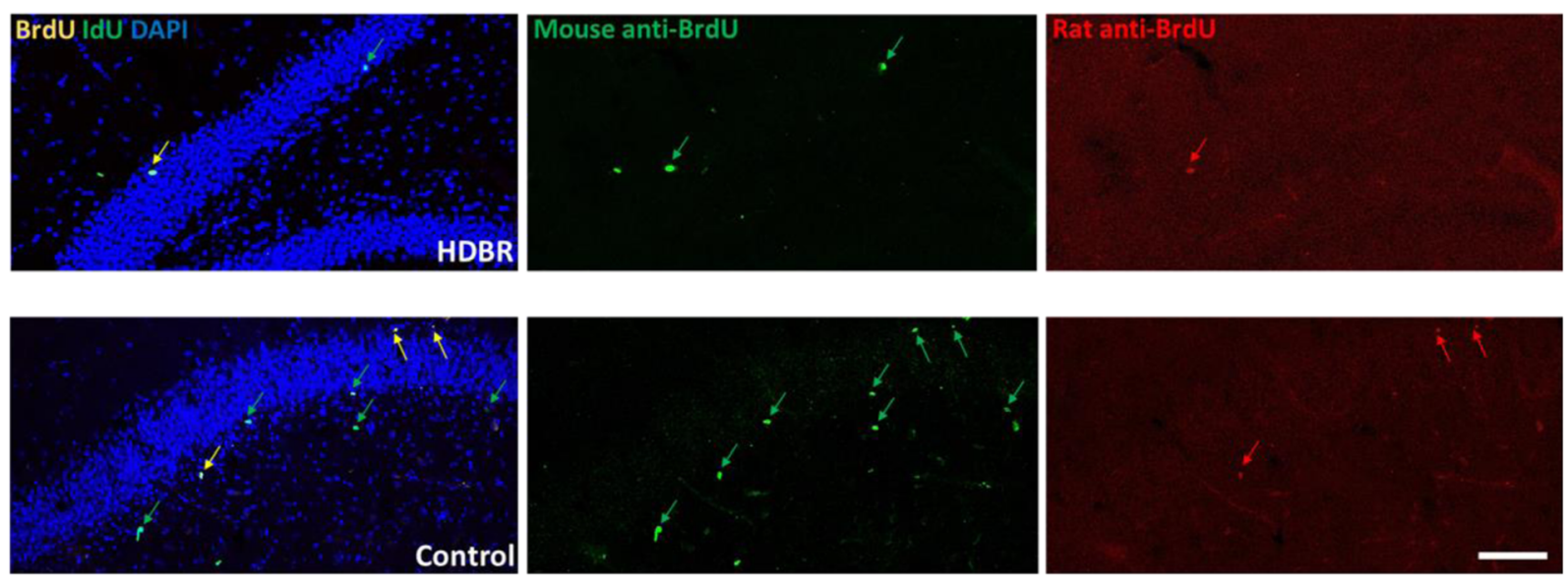

d

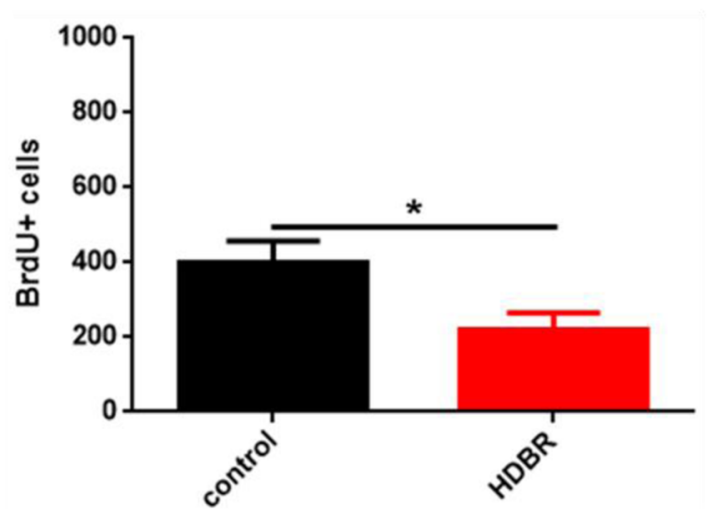

e

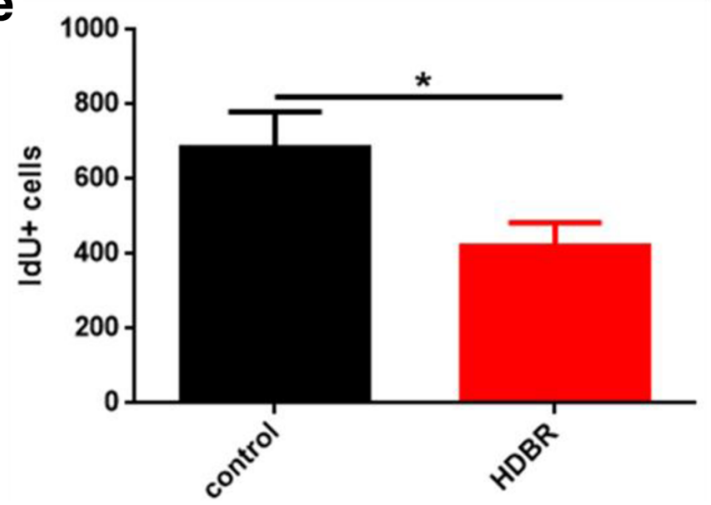

Fig. 1 HDBR impairs neurogenesis. a Schematic diagram of monkey in HDBR group. b Experimental procedure of immunohistochemistry. c Representative images of BrdU and IdU staining in HDBR (above) and control (below). d-e Quantitative results of immunofluorescence imaging of BrdU (d) and IdU (e) in the DG of HDBR and control monkeys. Data are shown as the mean \pm SEM. *P<0.05. Scale bar, 200um 
can test if simulated weightlessness could affect neurogenesis and speculate the effect of microgravity on the primate brain.

In this study, 5 monkeys were subjected to HDBR for 42 days to study the effects on the brain. Simultaneously, monkeys in the control group were single housed in the cages in the next room. While muscle atrophy and bone loss were also studied, those results are outside the scope of this paper. We collected tissue from the monkeys to examine whether HDBR for 42 days had an influence on adult hippocampal neurogenesis. Both BrdU (5-bromodeoxyuridin) and IdU (iododeoxyuridine) were intraperitoneally injected to label the newborn neurons. BrdU was injected before HDBR, while IdU was injected at the end of HDBR (Fig. 1b). Two primary antibodies, rat anti-BrdU (react with BrdU only) and mouse anti-BrdU(react with BrdU and IdU), were used for the separation of BrdU and IdU. BrdU-positive and IdU-positive cells in dentate gyrus (DG) were counted to represent the capacity of neurogenesis. We found significant reduction of cell survival by BrdU labeling (HDBR, $N=5$, control, $N=3$ ) and decreased cell proliferation by IdU labeling (HDBR, $\mathrm{N}=5$, control, $N=4$ ) in HDBR group compared with the control (Fig. 1c-e and Additional file 1: Table S1). These results demonstrated that 42 days HDBR impairs adult hippocampal neurogenesis.

Hippocampal neurogenesis has been observed in different adult animals. Studies have indicated that the newly generated cells might have a function in cognition and brain repair [12]. Adult hippocampal neurogenesis is also found in humans and contributes to memory function and enhanced synaptic plasticity across the life span. Adult hippocampal neurogenesis adds particular functionality to the mammalian hippocampus and presumably is involved in cognitive functions that we consider to be essential for humans [13]. Recent research found that recruitment of young neurons to the primate hippocampus decreases rapidly during the first years of life, and neurogenesis in the DG is extremely rare in adult [14], however, our results strongly prove that adult neurogenesis was still continued in adult monkeys.

During HDBR procedure, the monkeys were restrained on the bed. Several studies showed that volunteers developed psychic stress, and the plasm hormone involved in the response of the organism to stress, such as cortisol was significantly altered in human HDBR research [11]. Restraint stress has also been related to decreased cell proliferation and survival of the newborn hippocampal granule cells in mice. [15]. The decreased neurogenesis was caused by HDBR procedure, which might contain the effects from cephalic fluid shift and stress.

In conclusion, our results indicated the unambiguous neurogenesis in the adult rhesus macaque hippocampus, and simulated weightlessness HDBR procedure impairs the adult neurogenesis.

\section{Additional file}

Additional file 1: Table S1. Materials and Methods. Raw data of immunohistochemistry analysis. Figure S1. The Photo of monkey in HDBR group (DOCX $445 \mathrm{~kb}$ )

\section{Abbreviations}

BrdU: 5-bromodeoxyuridine; DG: Dentate gyrus; HDBR: Head-down bed rest; IdU: lododeoxyuridine; PBS: Phosphate buffered saline;

PFA: Paraformaldehyde

\section{Acknowledgments}

The authors are grateful to all those who participated in this research for their contributions.

\section{Funding}

This work was supported by, National Natural Science Foundation of China (NSFC) 91632103, Program of Shanghai Subject Chief Scientist 17XD1401700, Shanghai Education Commission Research and Innovation Program 2019-0107-00-02-E00037, "111" Program of Higher Education Discipline Innovation, "Eastern Scholar" (Shanghai Municipal Education Commission) and National Natural Science Foundation of Liaoning Province 201602233.

\section{Availability of data and materials}

All data generated or analyzed during this study are included in this article and Additional file1.

\section{Authors' contributions}

$W L$ and $X C^{*}$ designed the experiments. $X Z, X C^{\#}, L C$ and $W L$ wrote the article. All authors participated in the experiments or data analyze. All authors read and approved the final manuscript.

\section{Ethics approval}

All procedures were performed in accordance with the principles of the Association for Assessment and Accreditation of Laboratory Animal Care International (AAALAC), approved by Institutional Animal Care and Use Committee of China Astronaut Research and Training Center (ACC-IACUC2014-001) and Institutional Animal Care and Use Committee of Shanghai Jiao Tong University (IACUC-20140710).

Consent for publication

Not applicable.

Competing interests

The authors declare that they have no competing interests.

\section{Publisher's Note}

Springer Nature remains neutral with regard to jurisdictional claims in published maps and institutional affiliations.

\section{Author details}

${ }^{1}$ Bio-X Institutes, Key Laboratory for the Genetics of Development and Neuropsychiatric Disorders (Ministry of Education), Shanghai Key Laboratory of Psychotic Disorders, and Brain Science and Technology Research Center, Institute of Psychology and Behavioral Sciences, Shanghai Jiao Tong University, 800 Dongchuan Road, Shanghai 200240, China. ²Department of Obstetrics and Gynecology, the First Affiliated Hospital of Dalian Medical University, Dalian, China. ${ }^{3}$ National Key Laboratory of Human Factors Engineering, China Astronaut Research and Training Center, Beijing 100094, China. ${ }^{4}$ Department of Neurosurgery, Minhang Hospital, Fudan University, Shanghai, China.

Received: 12 March 2019 Accepted: 16 April 2019

Published online: 09 May 2019

\section{References}

1. Palinkas LA (2007) Psychosocial issues in long-term space flight: overview. Gravitational and Space Research 14. 
2. Koppelmans V, Erdeniz B, De Dios YE, Wood SJ, Reuter-Lorenz PA, et al. Study protocol to examine the effects of spaceflight and a spaceflight analog on neurocognitive performance: extent, longevity, and neural bases. BMC Neurol. 2013;13:205.

3. Lipnicki DM, Gunga HC, Belavy DL, Felsenberg D. Decision making after 50 days of simulated weightlessness. Brain Res. 2009;1280:84-9.

4. Karemaker JM, Berecki-Gisolf J. 24-h blood pressure in space: the dark side of being an astronaut. Respir Physiol Neurobiol. 2009;169:555-8.

5. Kawai Y, Doi M, Setogawa A, Shimoyama R, Ueda K, et al. Effects of microgravity on cerebral hemodynamics. Yonago Acta Med. 2003;46:1-8.

6. Li K, Guo X, Jin Z, Ouyang X, Zeng Y, et al. Effect of simulated microgravity on human brain gray matter and white matter--evidence from MRI. PLoS One. 2015;10:e0135835.

7. Hyde TM, Wu L-C, Krasnov IB, Sigworth SK, Daunton NG, et al. Quantitative autoradiographic analysis of muscarinic cholinergic and GABA a (benzodiazepine) receptors in the forebrain of rats flown on the soviet biosatellite COSMOS 2044. Brain Res. 1992;593:291-4.

8. Newberg AB. Changes in the central nervous system and their clinical correlates during long-term spaceflight. Aviation, space, and environmental medicine; 1994

9. Parimal C, Long A, Gabrielle H, Soulsby ME, Maxim D. Animal model of simulated microgravity: a comparative study of hindlimb unloading via tail versus pelvic suspension. Physiol Rep. 2013;1:e00012.

10. Ombergen AV, Demertzi A, Tomilovskaya E, Jeurissen B, Sijbers J, et al. The effect of spaceflight and microgravity on the human brain. J Neurol. 2017;264:18-22.

11. Choukèr A, Thiel M, Baranov V, Meshkov D, Kotov A, et al. Simulated microgravity, psychic stress, and immune cells in men: observations during 120-day 6 degrees HDT. J Appl Physiol. 2001;90:1736-43.

12. Hayashi F, Takashima N, Murayama A, Inokuchi K. Decreased postnatal neurogenesis in the hippocampus combined with stress experience during adolescence is accompanied by an enhanced incidence of behavioral pathologies in adult mice. Mol Brain. 2008;1:22.

13. Zhou M, Li W, Huang S, Song J, Kim JY, et al. mTOR inhibition ameliorates cognitive and affective deficits caused by Disc1 knockdown in adult-born dentate granule neurons. Neuron. 2013;77:647-54.

14. Sorrells SF, Paredes MF, Cebrian-Silla A, Sandoval K, Qi D, et al. Human hippocampal neurogenesis drops sharply in children to undetectable levels in adults. Nature. 2018:555:377.

15. Chu X, Zhou Y, Hu Z, Lou J, Song W, et al. 24-hour-restraint stress induces long-term depressive-like phenotypes in mice. Sci Rep. 2016;6:32935.

Ready to submit your research? Choose BMC and benefit from:

- fast, convenient online submission

- thorough peer review by experienced researchers in your field

- rapid publication on acceptance

- support for research data, including large and complex data types

- gold Open Access which fosters wider collaboration and increased citations

- maximum visibility for your research: over $100 \mathrm{M}$ website views per year

At $\mathrm{BMC}$, research is always in progress.

Learn more biomedcentral.com/submissions 